\title{
Erfahrungen mit dem Konzept des Kantons Zug für COVID-19
}

\section{Brian Martina ${ }^{a}$ Eva Martin-Diener ${ }^{b}$, Martin Pfister ${ }^{c}$, Rudolf Hauri ${ }^{d}$}

a PD Dr. med., bis Ende 2020 Kantonsarzt adjoint, Amt für Gesundheit Zug; Institut für Epidemiologie, Biostatistik und Prävention der Universität Zürich;

${ }^{\mathrm{b}} \mathrm{MPH}$, Institut für Epidemiologie, Biostatistik und Prävention der Universität Zürich; ${ }^{\mathrm{c}}$ Regierungsrat und Gesundheitsdirektor des Kantons Zug;

d Dr. med., Kantonsarzt, Amtsleiter Amt für Gesundheit Zug, Präsident Vereinigung der Kantonsärztinnen und Kantonsärzte der Schweiz VKS-AMCS

Zug hat ein öffentlich zugängliches Konzept für die Bekämpfung von COVID-19. Es wurde im Juli 2020 in der Schweizerischen Ärztezeitung vorgestellt und seither weiterentwickelt. Der Sommer und besonders die zweite Welle im Herbst 2020 haben die Möglichkeiten und Grenzen des Konzepts aufgezeigt.

Das im Juli 2020 [1] ausführlich beschriebene Alarmstufenkonzept ist ein Kernelement im Monitoringsystem des Kantons Zug für den COVID-19-Stab der Gesundheitsdirektion sowie das direktionsübergreifende
Unterstützungsgremium. Für dieses Monitoring werden mit verschiedenen Indikatoren die Fallzahlen für den Kanton Zug, die vier Nachbarkantone und die Schweiz aufbereitet, ausserdem für den Kanton Zug die
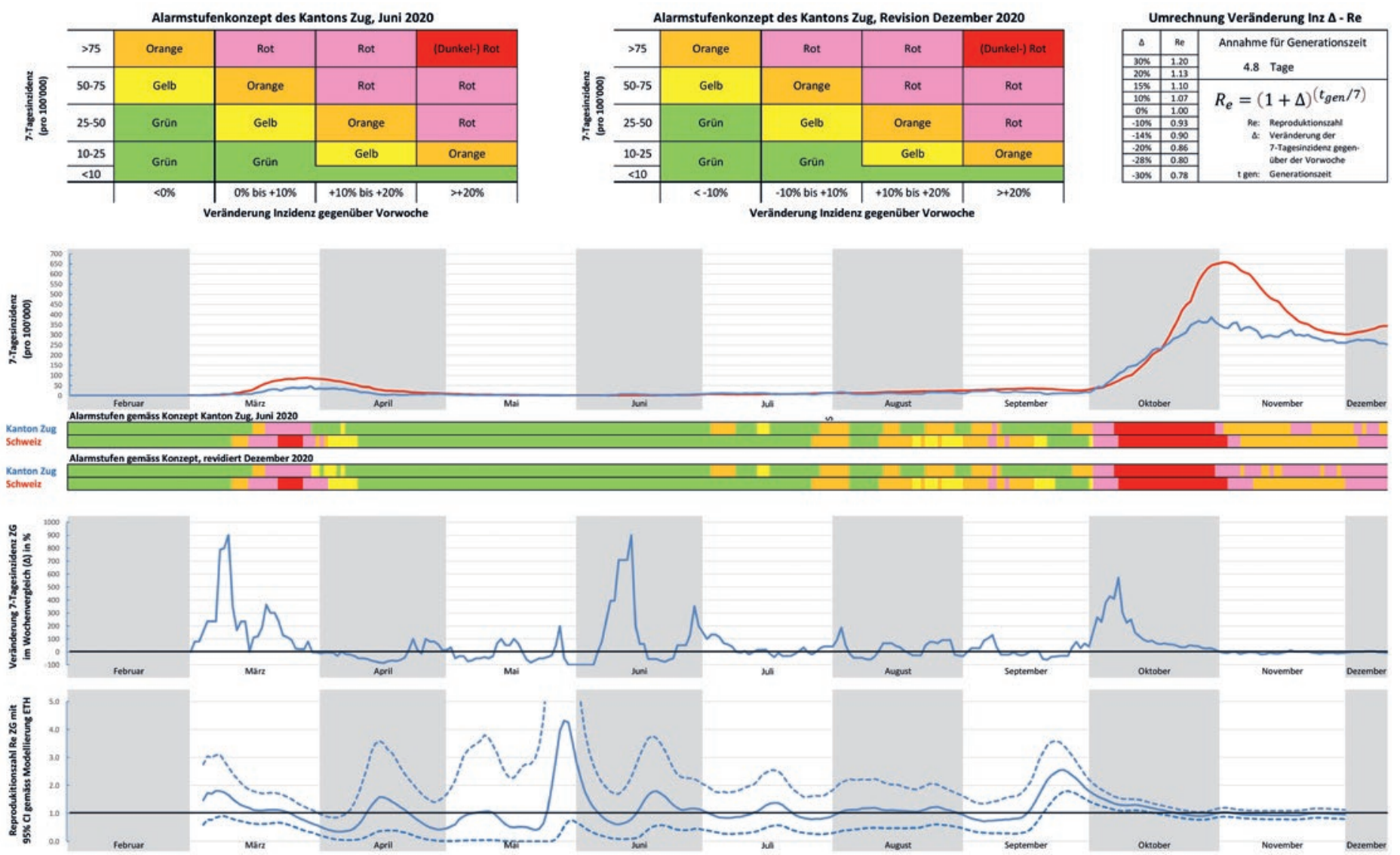

Abbildung 1: Entwicklung der 7-Tage-Inzidenzen und Alarmstufen für den Kanton Zug sowie die Schweiz und der Reproduktionszahl für Zug bis zum 10.12.2020. Das revidierte Alarmstufenmodell bringt den Handlungsbedarf angesichts der auf hohem Niveau stagnierenden Fallzahlen deutlich besser zum Ausdruck. Die Daten zur Reproduktionszahl (Re) entstammen der Website des Instituts für Integrative Biologie der ETH Zürich (heruntergeladen am 13.12.2020). Sie entsprechen im Wesentlichen einer um zwei Wochen verschobenen geglätteten Kurve der Änderungen der Inzidenz im Wochenvergleich. 
Zahlen zum Contact Tracing, zu den Hospitalisationen sowie zu den Todesfällen. Zusätzlich wurde für den Kanton der Verlauf der Veränderung der 7-Tage-Inzidenz im Wochenvergleich in das Monitoring aufgenommen. Dem Verlauf entspricht näherungsweise der zeitlich verschobene Verlauf der Reproduktionszahl (Re), die das etwa zwei Wochen vorher stattgefundene Ansteckungsgeschehen aufgrund der Fallmeldungen statistisch modelliert und die zunehmend als ein Element zur Beschreibung des Verlaufs der Pandemie verwendet wird (Abb. 1; alle Abb. online zu vergrössern). Diese Daten, zusammen mit einer Beschreibung des Contact Tracing sowie einer Zusammenfassung der Auswertungen der Befragungen der in den letzten sieben Tagen gemeldeten COVID-19-Fälle, sind die Grundlagen der kantonsärztlichen Lageeinschätzung, die seit Mitte September zweimal pro Woche veröffentlicht wird (Abb. 2). Details zum Monitoringsystem sowie zu den Erfahrungen damit und mit dem Contact Tracing sind in einem öffentlich zugänglichen Bericht der Gesundheitsdirektion Zug beschrieben [2].

\section{Der Verlauf in Zug bis Ende 2020}

Bereits ab Juni waren im Kanton Zug Episoden mit einem deutlichen relativen Anstieg der Inzidenz zu beobachten; zunächst waren die Fallzahlen allerdings noch auf sehr tiefem Niveau (Abb. 1). Ein erstes Maximum erreichte die 7-Tage-Inzidenz am 14. Juni mit 8/100 000, am gleichen Tag erreichte die Zunahme im
Wochenvergleich mit 900\% einen Extremwert. Am 3. Juli stieg die 7-Tage-Inzidenz mit erstmals über 10/100 000, dies bei einer Zunahme von 133\%. Damals wechselte die Alarmstufe für sechs Tage auf Orange. Gezielte Fallanalysen - inzwischen bezeichnet als Backward Tracing - konnten im Juni vermehrte Kontakte innerhalb einer erweiterten Familie und die wiedererwachende Partyszene sowie im Juli Rückkehrer aus Risikoländern in Südosteuropa und die inzwischen noch aktivere Ausgehszene als ursächliche Faktoren identifizieren. Beide Male konnten gezielte Massnahmen auf verschiedenen Ebenen die Situation wieder

Am Montag, 28. September, erfolgte für den Kanton Zug der Wechsel auf die Alarmstufe Orange, am Freitag auf Rot.

beruhigen. In der Folge gab es einige Phasen, während deren die Alarmstufe jeweils für wenige Tage auf Gelb oder Orange sowie für einen einzelnen Tag auf Rot wechselte.

Nachdem für den Kanton Zug zuvor 17 Tage die Alarmstufe Grün geherrscht hatte, erfolgte am Montag, dem 28. September, der Wechsel auf Orange, am Freitag auf Rot. Drei Tage später wechselte die Lageeinschätzung von «kontrolliert» auf «Situation mit erhöhter Aufmerksamkeit». An diesem Tag konnte auch letztmals eine systematische Auswertung aller gemeldeten Fälle durchgeführt werden. Am 6. Oktober fand in Zug eine Medienkonferenz statt, an der die besorgniserregende

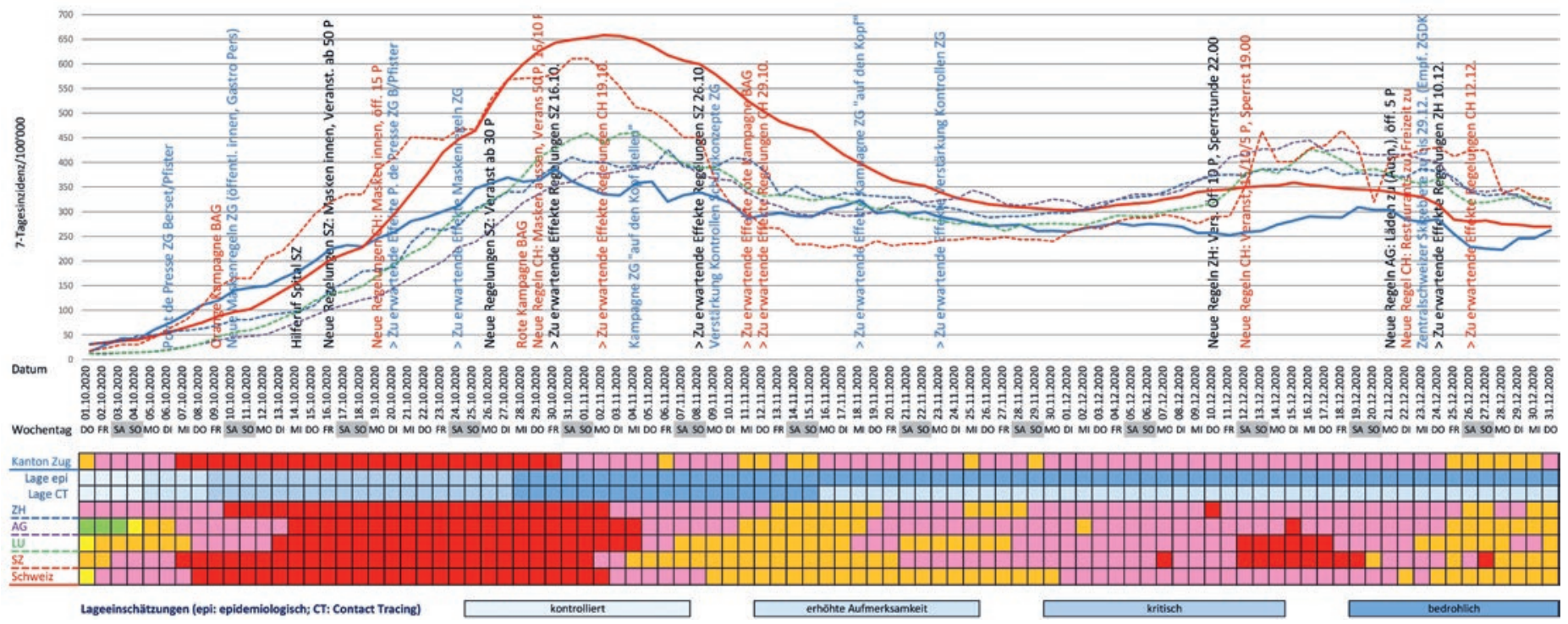

Abbildung 2: Entwicklung der 7-Tage-Inzidenzen und Alarmstufen in der Schweiz, im Kanton Zug und in den Nachbarkantonen von Anfang Oktober bis Ende Dezember 2020. Die Alarmstufen wurden aufgrund des im Dezember revidierten kantonalen Konzepts berechnet, das den erhöhten Handlungsbedarf bei einer Stagnation der Fallzahlen auf hohem Niveau besser zum Ausdruck bringt. Für den Kanton Zug sind zusätzlich die jeweiligen kantonsärztlichen Lageeinschätzungen dargestellt. Die den Berechnungen zugrundeliegenden nationalen Daten stammen von der Website des Bundesamts für Gesundheit BAG, die übrigen von den Websites der jeweiligen Kantone. 


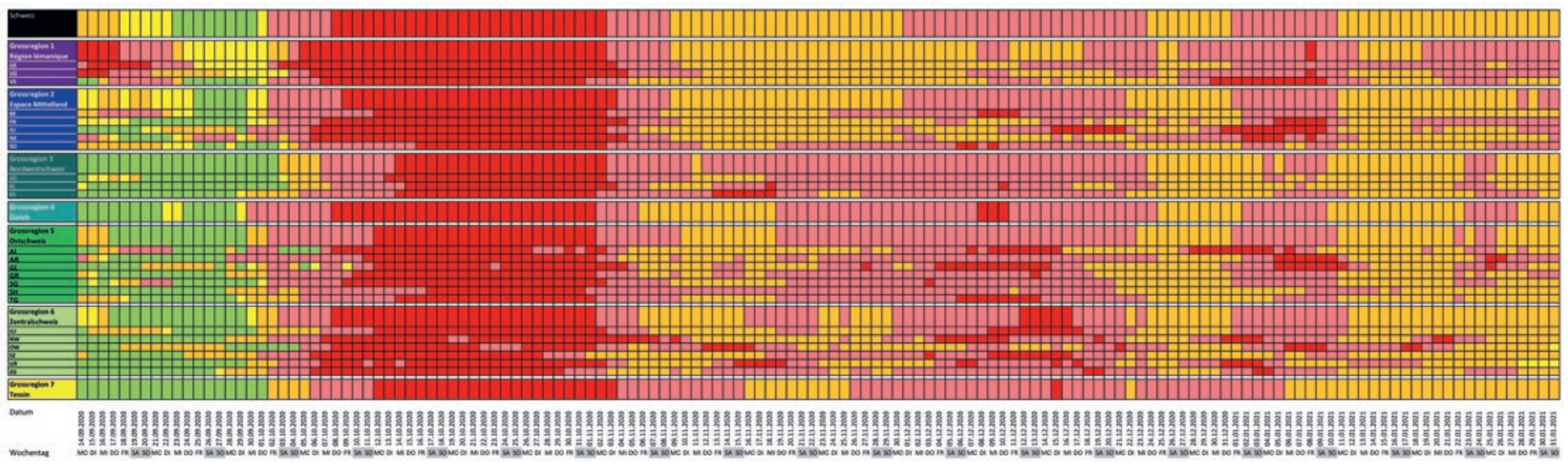

Abbildung 3: Entwicklung der Alarmstufen in der Schweiz, den Grossregionen sowie den Kantonen vom 14.9.2020 bis und mit 31.1.2021. Bei ansteigenden Fallzahlen geht das Konzept ab der Stufe Gelb von spezifischem Interventionsbedarf aus, ab Stufe Rot von der Notwendigkeit flächendeckender Massnahmen über die Kantone hinaus [1]. Von April 2020 bis Ende September hatte es nur auf Ebene einzelner Kantone oder Regionen mehrtägige Phasen in der Alarmstufe Rot oder Dunkelrot gegeben. Ab Anfang Oktober entwickelte sich aber eine solche Situation in allen Kantonen der Schweiz. Bis Ende Januar 2021 war es nur einzelnen Kanton gelungen, die 7-Tage-Inzidenz wieder unter 75/100000 zu senken und damit wieder die Alarmstufe Gelb zu erreichen. Die den Berechnungen zugrundeliegenden Fallzahlen stammen vom Bundesamt für Gesundheit. In seiner Lagebeurteilung zu Covid-19 liefert der Koordinierte Sanitätsdienst KSD seinen Partnern unter anderem eine aktualisierte Übersicht zu den Alarmstufen für die Grossregionen und die Kantone. Entsprechend seiner etablierten Farbgebung hat er dabei die Stufe Rot durch Dunkelorange und die Stufe Dunkelrot durch Rot ersetzt.

Entwicklung vom kantonalen Gesundheitsdirektor und vom zuständigen Bundesrat thematisiert wurde. Am 7. Oktober wechselte die Alarmstufe auf Dunkelrot, zwei Tage später wurde die Lage als «kritisch» eingeschätzt.

Die prozentuale Zunahme der Inzidenz schwächte sich daraufhin kontinuierlich ab, so dass bis zum 27. Oktober im Wesentlichen eine lineare Zunahme der 7-TageInzidenz zu beobachten war (Abb. 2). Diese erreichte am 30. Oktober mit 386/100 000 ein Maximum für den Kanton Zug. Es folgte zunächst eine mässige Abnahme der Inzidenz, dann eine Stagnation auf hohem Niveau. Gemäss dem Alarmstufenkonzept des Kantons Zug wechselte die nationale Situation ebenfalls am 2. Oktober auf Rot und am 8. Oktober - nach Berücksichtigung der Nachmeldungen - auf Dunkelrot. Der Anstieg der Inzidenz erfolgte zunächst etwas langsamer, dann aber schneller und länger als in Zug, das Maximum von 658/100 000 wurde am 2. November erreicht. Im Nachbarkanton Schwyz erfolgte ein deutlich rascherer Anstieg als in Zug, die maximale Inzidenz betrug 610. In den Nachbarkantonen Zürich, Aargau und Luzern erfolgte der Anstieg später, dann aber etwas stärker und länger als in Zug.

\section{Erfahrungen und Konsequenzen}

Alarmstufenkonzept, Monitoringdokumentation und Lageeinschätzungen haben sich für den Kanton Zug grundsätzlich bewährt. Die Transparenz über die verwendeten Indikatoren und Beurteilungskriterien haben vertrauensbildend gegenüber Gemeinden und anderen Partnern gewirkt und eine faktenbezogene Diskussion über Sinn, Notwendigkeit und Schwerpunkte beispielsweise der Kontrolle der Schutzkonzepte ermöglicht. In diesen Gesprächen war es nie ein Problem zu vermitteln, dass Alarmstufen stets in ihrem mehrtägigen Verlauf zu beurteilen sind und dass sie zudem nur mit Anhaltspunkten, nicht aber mit Automatismen bezüglich bevölkerungsbezogener Massnahmen verbunden sein können. Interessanterweise hat das Zuger Konzept auch die öffentliche Diskussion in anderen Kantonen belebt, beispielsweise als die Presse Ende August aufgrund eigener Berechnungen feststellte, dass ein Nachbarkanton sich bereits in Alarmstufe Rot befand. Dieser Kanton übernahm in der Folge wesentliche Teile der in Zug entwickelten Konzepte und Handlungsoptionen.

\section{Das Alarmstufenkonzept hat sich bewährt, die} zweite Welle zeigte aber seine Limitationen auf.

Der Verlauf der zweiten Welle der COVID-19-Pandemie zeigte aber auch die Limitationen des Alarmstufenkonzepts auf. Aufgrund der Erfahrungen des Frühlings 2020 war nicht mit einer Situation gerechnet worden, in der die Fallzahlen über längere Zeit auf hohem Niveau stagnieren könnten. Die dadurch entstehenden Gewöhnungs- und Normalisierungseffekte in der Öffentlichkeit und im politischen Diskurs, die Be- und Überlastung des Gesundheitswesens, der Gesundheitsbehörden, aber auch anderer Teile der Gesellschaft sowie die Zunahme sozialer Spannungen haben deutlich gemacht, dass auch eine solche Situation mit einem 
erhöhten Handlungsbedarf verbunden ist. Dieser erhöhte Handlungsbedarf wurde in einer Revision des Alarmstufenkonzepts im Dezember 2020 berücksichtigt: auf dem höchsten Inzidenzniveau ist für ein Wechsel von der Alarmstufe Rot auf Orange nun ein Rückgang der 7-Tage-Inzidenz von mindestens 10\% notwendig (Abb. 1), dies in Anlehnung an das Vorgehen des European Center for Disease Prevention and Control ECDC bei Ländervergleichen [2]. Abbildung 3 zeigt eine Anwendung des revidierten Alarmstufenkonzepts auf die Grossregionen und die Kantone der Schweiz.

Entscheidungen zu bevölkerungsbezogenen Massnahmen wurden in der zweiten Welle in erster Linie durch die Kantone, dann aber auch durch den Bund getroffen. Diese Entscheidungen erfolgten generell mit einer grösseren Verzögerung zum Fallgeschehen als in der ersten Welle. Der Kanton Zug hat mit der erwähnten Pressekonferenz am 6. Oktober sowie mit den am 5. Oktober durch den Regierungsrat beschlossenen und am 10. Oktober in Kraft getretenen verschärften Maskenregeln relativ schnell auf die Entwicklung reagiert. Offenbar wären für eine Trendumkehr weitgehendere und überkantonal abgestimmte Massnahmen nötig gewesen. Vielleicht hat aber die Reaktion des Kantons Zug doch zu einem etwas günstigeren Verlauf als in den Nachbarkantonen beigetragen (Abb. 2).

Das Alarmstufenkonzept geht implizit davon aus, dass eine 7-Tage-Inzidenz von unter 10/100 000 anzustreben ist. Diese Annahme entspricht auch einem inzwischen erfolgten Aufruf internationaler Expertinnen und Experten in der Fachzeitschrift Lancet, der europaweit das Ziel von maximal 10 neuen Fällen pro Million Einwohner täglich postuliert [3]. Zumindest bis zu einer weitgehenden Durchimpfung der vulnerablen Bevölkerungsgruppen scheint diese Zielsetzung weiterhin sinnvoll, angesichts des Zusammenhangs zwi- schen Fallzahlen und dem Risiko für das Auftauchen neuer Virus-Varianten allenfalls sogar darüber hinaus. Das Konzept kann auch als Orientierungshilfe im Hinblick auf die Lockerung von Massnahmen dienen. So kann von deren nachhaltiger Wirksamkeit wohl kaum ausgegangen werden, bevor die 7-Tage-Inzidenz nicht deutlich unter 50/100 000 fällt, was einer Alarmstufe Grün über mindestens eine Woche entspricht. Ein Zurückfahren der Massnahmen auf das Niveau des Sommers 2020 erscheint erst bei einer Stabilisierung unter 10/100000 und damit nach einer längerfristigen «Phase Grün» sinnvoll. Die Erfahrungen des Herbstes 2020 haben gezeigt, dass dabei Kantone nicht isoliert, sondern im Verbund betrachtet werden müssen, im Hinblick auf nationale Massnahmen wohl über Regionen hinweg.

Die Herausforderungen bezüglich der Abstimmung zwischen Kantonen und Bund sowie die Notwendigkeit eines raschen und entschlossenen Handelns sind in der zweiten Welle deutlich geworden. Ein transparentes und nachvollziehbares Vorgehen kann die öffentliche und die politische Diskussion versachlichen und hoffentlich auch zu kürzeren Entscheidungswegen beitragen.

\section{Literatur}

1 Martin B, Klaey H, Müller A, Gügler C, Koepfli A, Gross B, Pfister M, Hauri R. Das Konzept des Kantons Zug für die zweite Welle von COVID-19. Schweiz Ärzteztg. 2020;101(31-32):928-32. doi.org/10.4414/saez.2020.19087

2 Martin B, Martin-Diener E, Pfister M, Hauri R. Erfahrungen mit dem Konzept des Kantons Zug für COVID-19 bis Ende 2020 Zug, Gesundheitsdirektion 2021. www.zg.ch/corona

3 European Centre for Disease Prevention and Control (ecdc). Covid-19 country overviews. Heruntergeladen am 14.12.2020 von www.covid19-country-overviews.ecdc.europa.eu

4 Priesemann V, Brinkmann MM, Ciesek S, et al. Calling for panEuropean commitment for rapid and sustained reduction in SARS-CoV-2 infections. Lancet. 2021;397(10269):92-3. doi.org/10.1016/S0140-6736(20)32625-8
PD Dr. med. Brian Martin,

$\mathrm{MPH}$

Armeestab-Sanität

Worblentalstrasse 36

CH-3063 Ittigen

Tel. 0584855463

brian.martin[at]vtg.admin.ch

\section{Das Wichtigste in Kürze}

- Das Konzept des Kantons Zug zur Bekämpfung von COVID-19 hat sich grundsätzlich bewährt. Die Transparenz über die verwendeten Indikatoren und Beurteilungskriterien wirkten vertrauensbildend und haben eine faktenbezogene Diskussion derThematik ermöglicht.

- In der zweiten Pandemiewelle stagnierten die Fallzahlen über längere Zeit auf hohem Niveau, womit nicht gerechnet worden war. Die Überlastung des Gesundheitswesens und der Gesundheitsbehörden hat gezeigt, dass diese Situation mit einem erhöhten Handlungsbedarf verbunden ist. Daher wurde das Alarmstufenkonzept im Dezember 2020 revidiert. - Das Zuger Konzept kann auch Orientierung in Bezug auf die Lockerung von Massnahmen bieten. Die bisherigen Erfahrungen mit COVID-19 haben gezeigt, dass Kantone nicht isoliert, sondern im Verbund betrachtet werden müssen.

\section{L'essentiel en bref}

- Le concept du canton de Zoug pour lutter contre le COVID-19 a fait ses preuves. La transparence des indicateurs et des critères d'évaluation utilisés ont permis de renforcer la confiance et d'entamer une discussion factuelle sur la question.

- $\quad$ Lors de la deuxième vague de la pandémie, le nombre de cas a stagné à un niveau élevé pendant longtemps, ce qui n'était pas prévu. Système de santé surchargé et autorités sanitaires débordées: I'urgence de la situation a montré qu'il fallait impérativement agir. Le concept de niveau d'alerte a donc été révisé en décembre 2020.

- Le concept zougois peut également servir de point de repère lorsqu'il s'agira d'assouplir les mesures. L'expérience acquise jusqu'à présent avec le COVID-19 a montré que les cantons ne doivent pas être pris en compte séparément, mais comme un tout. 